\title{
Shannon, Tsallis and Kaniadakis Entropies in Bi- level Image Thresholding
}

\section{Amelia Carolina Sparavigna'}

${ }^{1}$ Department of Applied Science and Technology, Politecnico di Torino, Italy

Abstract: The maximum entropy principle is often used for bi-level or multi-level thresholding of images. For this purpose, some methods are available based on Shannon and Tsallis entropies. In this paper, we discuss them and propose a method based on Kaniadakis entropy.

Keywords: Kaniadakis Entropy, Image Processing, Image Segmentation, Image Thresholding, Texture Transitions

\section{Introduction}

The concept of entropy was born in thermodynamics and statistical mechanics. Shannon, in 1948, formulated it for the theory of information, obtaining the "information entropy". In an intuitive understanding of it [1], this entropy relates to the amount of uncertainty about an event associated with a given probability distribution.

In image processing, Shannon entropy was the first being used, but today, it is the Tsallis formulation of entropy that seems to be preferred [2-4]. For the elaboration of images, the entropy uses their histograms. For instance, in the bi-level segmentation of a gray-level image, a threshold is determined which separates the gray tones in two systems $A$ and $B$, maximizing the entropy. Considering $A$ and $B$ independent, the entropy $S(A \cup B)$ is the generalized sum $S^{A} \oplus S^{B}$, where $S^{A}, S^{B}$ are the corresponding entropies of the systems. In this paper, we will discuss the use of Shannon and Tsallis entropies for image thresholding. Among the other formulations of entropy [5], here we propose the thresholding using Kaniadakis entropy, which is a quite attractive entropy based on the relativistic formulation of the statistical mechanics $[6,7]$.

Both Tsallis and Kaniadakis entropies have an entropic index. If these entropies are used for a bi-level thresholding of an image, the bi-level black and white image that we obtain depends on the value of the optimize threshold, which is depending on the entropic index. Here we compare the results we can obtain with Kaniadakis and Tsallis entropies, proposing a "measure" on the output image. In this "measure", we evaluate the number of edge pixels that separate black and white regions. After experiments on some images, we can conclude that the two entropies compare positively. We have the same results, but Kaniadakis entropy has the intuitive advantage of recovering the Shannon result when its entropic index goes to zero.

\section{Images and information entropy}

Before proposing the use of Kaniadakis entropy for image thresholding, let us shortly illustrate the classic method using the Shannon entropy applied for segmentation. Let us consider an image which as $N$ elements of luminance. These elements can have $g$ gray tones, labelled $\left\{x_{1}, x_{2}, \ldots, x_{n}\right\}$, for instance $\{0,1,2, \ldots, g\}$, with $g=255$. Let us suppose that each tone $x_{i}$ is chosen $N_{i}$ times. The frequency is $f_{i}=N_{i} / N$. Accordingly, we have a set $F=$ $\left\{f_{1}, f_{2}, \ldots, f_{n}\right\}$, that we call "the scene". Any given scene has a certain multiplicity $W_{F}$ :

$W_{F}=\frac{N !}{\left(N f_{1}\right) ! \ldots\left(N f_{n}\right) !}$

Equation (1) gives the number of ways in which we can generate the same scene [8]. Let us consider that each of the generated copy of the scene has the same probability, which is equal to $1 / W_{F}$. In the case $N$ is large, we can apply the Stirling approximation: 


$$
\begin{aligned}
& \frac{1}{N} \ln W_{F}=\frac{1}{N} \ln \left(\frac{N !}{\left(N f_{1}\right) ! \ldots\left(N f_{n}\right) !}\right)=\frac{1}{N}\left\{\ln N !-\left[\ln \left(N f_{1}\right) !+\ldots+\ln \left(N f_{n}\right) !\right]\right\} \\
& =\frac{1}{N} N \ln N-N-\frac{1}{N}\left[N f_{1} \ln N f_{1}+N f_{1}+\ldots+N f_{n} \ln N f_{n}+N f_{n}\right] \\
& =\frac{1}{N} N \ln N-\frac{1}{N}\left[N f_{1} \ln N+N f_{1} \ln f_{1}+\ldots+N f_{n} \ln N+N f_{n} \ln f_{n}\right] \\
& =-\left[f_{1} \ln f_{1}+\ldots+f_{n} \ln f_{n}\right]=-\sum_{i=1}^{n} f_{i} \ln f_{i}
\end{aligned}
$$

In (2), we use $\sum_{i=1}^{n} f_{i}=1$. This is the Shannon entropy; it is depending on "objective" frequencies $f_{i}$ instead of "subjective" probabilities $p_{i}[8]$.

\section{Thresholding}

For a bi-level thresholding of an image, let us follow the approach of reference 4.

Let us consider two independent systems $A$ and $B$, for which the joint probability is $p(A, B)=p(A) p(B)$. The entropy $S(A \cup B)$ is $S^{A} \oplus S^{B}$. The systems can be given as in the following [4]. A contains elements with $g$ gray tones, labelled $\left\{x_{1}, x_{2}, \ldots, x_{t}\right\}$, for instance $\{0,1,2, \ldots, t\}$, that is, with gray tone below or equal a given threshold $t$. Let us suppose that each tone $x_{i}$ is chosen $N_{A, i}$ times, according to frequency: $f_{A, i}=f_{i} N / N_{A}$. We have: $\quad N_{A}=\sum_{i=1}^{t} N f_{i}=N \sum_{i=1}^{t} f_{i}=N P_{A}$

Therefore: $\quad f_{A, i}=N f_{i} /\left(N P_{A}\right)=f_{i} / P_{A}$

Moreover, $\sum_{i=1}^{t} f_{A, i}=1$. In the same manner, $B$ contains elements $\left\{x_{t+1}, x_{2}, \ldots, x_{n}\right\}$, that is, $\{t+1, t+2, \ldots, g\}$. Let us suppose that each tone $x_{i}$ is chosen $N_{B, i}$ times, according to frequency: $f_{B, i}=f_{i} N / N_{B}$

Again:

$N_{B}=\sum_{i=t+1}^{g} N f_{i}=N \sum_{i=t+1}^{g} f_{i}=N P_{B}$.

The entropy $S^{A}$ is:

Then, using Stirling again:

$$
S^{A}=\frac{1}{N_{A}} \ln W_{A}=\frac{1}{N_{A}} \ln \left(\frac{N_{A} !}{\left(N_{A, 1}\right) ! \ldots\left(N_{A, t}\right) !}\right)
$$

For B:

$$
S^{A}=-\sum_{i=1}^{t} f_{A, i} \ln f_{A, i}=-\sum_{i=1}^{t} \frac{f_{i}}{P_{A}} \ln \frac{f_{i}}{P_{A}}
$$

$$
S^{B}=\frac{1}{N_{B}} \ln W_{B}=\frac{1}{N_{B}} \ln \left(\frac{N_{B} !}{\left(N_{B, t+1}\right) ! \ldots\left(N_{B, g}\right) !}\right)=-\sum_{i=t+1}^{g} \frac{f_{i}}{P_{B}} \ln \frac{f_{i}}{P_{B}}
$$

The abovementioned frequencies for a gray-level image can be given by the normalized histogram.

To find the best value of threshold t, we have to maximize $S^{A}+S^{B}$ [4].

\section{Bi-level thresholding with Tsallis and Kaniadakis entropies}

Let us remember that the Tsallis entropy is given by $[9,10]$ :

$$
S_{q}=\frac{1}{q-1}\left\{1-\sum_{i} p_{i}^{q}\right\}=\frac{1}{q-1}\left\{\sum_{i} p_{i}\left(1-p_{i}^{q-1}\right)\right\}
$$

In fact, the Tsallis entropy is defined, using the $q$-logarithm, as: 


$$
S_{q}=-\sum_{i} p_{i}^{q} \ln _{q} p_{i}=-\sum_{i} p_{i}^{q} \frac{p_{i}^{1-q}-1}{1-q}=-\sum_{i} \frac{p_{i}-p_{i}^{q}}{1-q}=\frac{1}{q-1}\left\{1-\sum_{i} p_{i}^{q}\right\}
$$

Let us assume a bi-level threshold $t$ for the gray levels. In [3], two systems had been introduced, $A$ and $B$, and their probability distributions. Let us assume the properties of $A$ and $B$ as in the previous section. The Tsallis entropies, one for each distribution, are given by:

$$
\begin{aligned}
& S_{q}^{A}(t)=\frac{1}{q-1}\left\{1-\sum_{i=1}^{t}\left(\frac{f_{i}}{P_{A}}\right)^{q}\right\} \\
& S_{q}^{B}(t)=\frac{1}{q-1}\left\{1-\sum_{i=t+1}^{g}\left(\frac{f_{i}}{P_{B}}\right)^{q}\right\}(9)
\end{aligned}
$$

Taking the limit $q \rightarrow 1$, Tsallis entropy gives Shannon's entropy. The total Tsallis entropy is given by the generalized sum:

$$
S_{q}(t)=S_{q}^{A}(t)+S_{q}^{B}(t)+(1-q) S_{q}^{A}(t) S_{q}^{B}(t)
$$

In fact, in a generalization of statistical mechanics, a deformed entropy had been proposed, the Kaniadakis entropy, also known as א-entropy [6,7]:

$$
S_{\kappa}=-\sum_{i} p_{i} \ln _{\{\kappa\}}\left(p_{i}\right)
$$

This entropy has the remarkable property of having the same behavior of Shannon entropy, that is:

$$
S_{\kappa}=\sum_{i} p_{i} \ln _{\{\kappa\}}\left(\frac{1}{p_{i}}\right)
$$

In it, we have the generalized version of the logarithm [7]:

$$
S_{\kappa}=-\frac{1}{2 \kappa}\left\{\sum_{i}\left(\left(p_{i}\right)^{1+k}-\left(p_{i}\right)^{1-k}\right)\right\}
$$

We can apply this entropy to the bi-level thresholding. Let us call the threshold $\tau$.

Generalizing (3) and (5), $\kappa$-entropies are:

$$
\begin{aligned}
& S_{\kappa}^{A}(\tau)=-\frac{1}{2 \kappa}\left\{\sum_{i=1}^{\tau}\left(\left(\frac{f_{i}}{P_{A}}\right)^{1+k}-\left(\frac{f_{i}}{P_{A}}\right)^{1-k}\right)\right\} \\
& S_{\kappa}^{B}(\tau)=-\frac{1}{2 \kappa}\left\{\sum_{i=\tau+1}^{g}\left(\left(\frac{f_{i}}{P_{B}}\right)^{1+k}-\left(\frac{f_{i}}{P_{B}}\right)^{1-k}\right)\right\}
\end{aligned}
$$

In the limit $\kappa \rightarrow 0$, Kaniadakis entropy becomes Shannon entropy.

Let us consider the composition of systems $A$ and $B$, but in the framework of this deformed statistics. According to [11], the generalized sum:

$$
S_{\kappa}(\tau)=S_{\kappa}^{A}(\tau) \mathfrak{J}_{\kappa}^{B}(\tau)+S_{\kappa}^{B}(\tau) \mathfrak{J}_{\kappa}^{A}(\tau)
$$

In (16) we have:

$$
\begin{array}{r}
\mathfrak{J}_{\kappa}^{A}(\tau)=\frac{1}{2}\left\{\sum_{i=1}^{\tau}\left(\left(\frac{f_{i}}{P_{A}}\right)^{1+k}+\left(\frac{f_{i}}{P_{A}}\right)^{1-k}\right)\right\} \\
\mathfrak{I}_{\kappa}^{B}(\tau)=\frac{1}{2}\left\{\sum_{i=\tau+1}^{g}\left(\left(\frac{f_{i}}{P_{B}}\right)^{1+k}+\left(\frac{f_{i}}{P_{B}}\right)^{1-k}\right)\right\}
\end{array}
$$


When entropies, (10) or (16), are maximized, the corresponding gray level $\tau$ is considered the optimum threshold value. In the gray bi-level thresholding, we have a resulting processed image, which is a black and white image. The output image is created as in the following: if pixels have a gray tone larger than the threshold, they become white. If pixels have a lower value, they become black.

\section{More deeply in the limit of Kaniadakis entropy}

In fact, we have that:

$$
\mathfrak{J}_{\kappa}=\frac{1}{2}\left\{\sum_{i} p_{i}^{1+\kappa}+\sum_{i} p_{i}^{1-\kappa}\right\}=\kappa S_{\kappa}+\sum_{i} p_{i}^{1+\kappa}
$$

That is:

$$
\begin{aligned}
& \mathfrak{I}_{\kappa}=\frac{1}{2}\left\{\sum_{i} p_{i}^{1+\kappa}+\sum_{i} p_{i}^{1-\kappa}\right\}=-\kappa \frac{1}{2 \kappa}\left\{\sum_{i} p_{i}^{1+\kappa}-\sum_{i} p_{i}^{1-\kappa}\right\}+\sum_{i} p_{i}^{1+\kappa} \\
& =-\frac{1}{2} \sum_{i} p_{i}^{1+\kappa}+\frac{1}{2} \sum_{i} p_{i}^{1-\kappa}+\sum_{i} p_{i}^{1+\kappa}=\mathfrak{J}_{\kappa}
\end{aligned}
$$

Therefore:

$$
\begin{aligned}
& S_{\kappa}=S_{\kappa}^{A} \mathfrak{J}_{\kappa}^{B}+S_{\kappa}^{B} \Im_{\kappa}^{A} \\
& =S_{\kappa}^{A}\left(\kappa S_{\kappa}^{B}+\sum_{i=\tau+1}^{g}\left(\frac{f_{i}}{P_{B}}\right)^{1+\kappa}\right)+S_{\kappa}^{B}\left(\kappa S_{\kappa}^{A}+\sum_{i=1}^{\tau}\left(\frac{f_{i}}{P_{A}}\right)^{1+\kappa}\right) \\
& S_{\kappa}=2 \kappa S_{\kappa}^{A} S_{\kappa}^{B}+S_{\kappa}^{A} \sum_{i=\tau+1}^{g}\left(\frac{f_{i}}{P_{B}}\right)^{1+\kappa}+S_{\kappa}^{B} \sum_{i=1}^{\tau}\left(\frac{f_{i}}{P_{A}}\right)^{1+\kappa}
\end{aligned}
$$

In the limit $\kappa \rightarrow 0$, Kaniadakis entropy becomes Shannon entropy, and therefore we must have the normal additivity. And in fact:

$$
S_{\kappa}=S^{A} \sum_{i=\tau+1}^{g} \frac{f_{i}}{P_{B}}+S^{B} \sum_{i=1}^{\tau} \frac{f_{i}}{P_{A}}=S^{A}+S^{B}
$$

$S^{A}, S^{B}$ are the Shannon entropies. In the limit $\sum_{i=1}^{\tau}\left(\frac{f_{i}}{P_{A}}\right)^{1+\kappa}$ becomes $\sum_{i=1}^{\tau} \frac{f_{i}}{P_{A}}=\frac{1}{P_{A}} \sum_{i=1}^{\tau} f_{i}=\frac{P_{A}}{P_{A}}=1$. We have the same for $B$.

\section{Discussion}

Let us note that, both Tsallis and Kaniadakis entropies have entropic indices that can give different results when applied to the sample. To choose among these several results and define an output image, we propose a "measure" of the bi-level image, given by the number of edge pixels between black and white regions. We imagine that, increasing the number of edge pixels, we are able to see more textures in the black and white image. Of course, other measures can be defined.

Figures 1-5 give images, bi-level images and the corresponding Tables, which are displaying the results of maximizing Tsallis and Kaniadakis entropies, according to (10) and (16). In experiments, their entropic indices are spanning interval $(0,1)$. Let us avoid, in the calculations, the values 0 and 1 .

Of the five examples proposed in the corresponding figures, we have that the results we obtain using the
Kaniadakis entropy are the same of that we obtain with Tsallis entropy, within an uncertainty of one gray-tone. Let us also note that, in three of the examples, the best result that we obtain, according to the proposed "measure", is the same of that we can have from the Shannon approach. However, in the case of the two examples (Figs. 4 and 5), where the method is applied to microscopic images of blood, the best result is different from that given by the Shannon limit. Moreover, in the case of Figure 5, we have the evidence of an "image transition" (see Ref.12 for more details).

Besides the fact that the Kaniadakis entropy possesses a formalism closer to that of Shannon entropy, a good reason for preferring $\kappa$-entropy is that it has the more intuitive behavior of an entropy recovering the Shannon result, when its entropic index is going to zero. Another relevant advantage of Kaniadakis entropy is in the evaluation of multi-level thresholding 
of images. This will be discussed in a future paper.

\section{References}

1. Sonka, M.; Hlavac, V.; Boyle, R. Image processing, analysis, and machine vision, Cengage Learning, 2014. http://dx.doi.org/10.1007/978-1-4899-3216-7

2. Gull, S.F.; Skilling, J. Maximum entropy method in image processing. Communications, Radar and Signal Processing, IEE Proceedings F, 1984, 131, 646-659. http://dx.doi.org/10.1049/ip-f-1.1984.0099

3. Portes de Albuquerque, M.; Esquef, I.A.; Gesualdi Mello, A.R.; Portes de Albuquerque, M. Image thresholding using Tsallis http://dx.doi.org/10.1016/j.patrec.2004.03.003

4. Kapur, J.N.; Sahoo, P.K.; Wong, A.K.C. A new method for gray-level picture thresholding using the entropy of the histogram. Comput. Vision Graphics Image Process., 1985, 29, 273-285. http://dx.doi.org/10.1016/0734-189x(85)90125-2

5. Beck, C. Generalised information and entropy measures in physics, Contemporary Physics, 2009, 50, 495-510. http://dx.doi.org/10.1080/00107510902823517
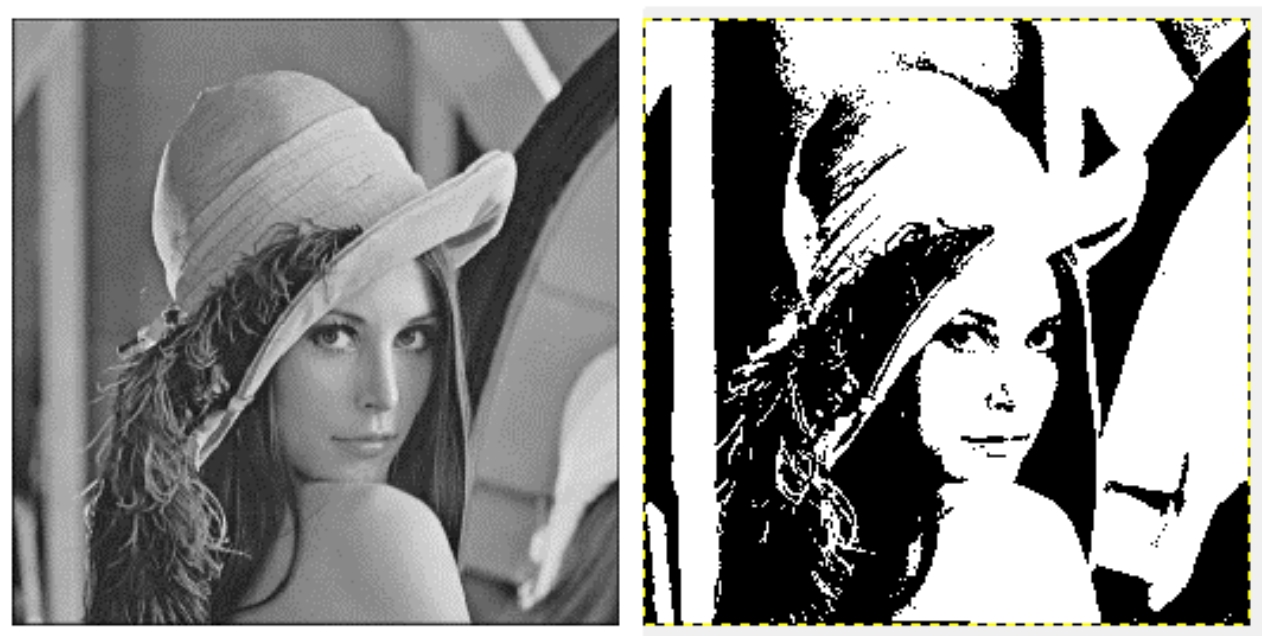

$\mathbf{t}=\mathbf{1 2 0}$ http://dx.doi.org/10.1103/physreve.66.056125 http://dx.doi.org/10.3390/e15103983 86 http://dx.doi.org/10.1103/physreve.72.026123 entropy. Pattern Recognition Letters, 2004, 25, 1059-1065.

6. Kaniadakis, G. Statistical mechanics in the context of special relativity, Phys. Rev. E, 2002, 66, 056125.

7. Kaniadakis, G. Theoretical foundations and mathematical formalism of the power-law tailed statistical distributions, Entropy, 2013, 15, 3983-4010.

8. Jaynes, E.T. Where do we go from here?, in C. Ray Smith and W.T. Grandy, Jr. (eds.), Maximum-entropy and Bayesian methods in inverse problems, 21-58, 1985, D. Reidel Publishing Company. http://dx.doi.org/10.1007/978-94-017-2221-6_2

9. Tsallis, C. Introduction to nonextensive statistical mechanics, 2009, Springer. http://dx.doi.org/10.1007/978-0-387-85359-

10. Yamano, T. Some properties of q-logarithm and q-exponential functions in Tsallis statistics, Physica A 2002, 305, 486-496. http://dx.doi.org/10.1016/s0378-4371(01)00567-2

11. Scarfone, A.M.; Wada, T. Thermodynamic equilibrium and its stability for microcanonical systems described by the SharmaTaneja-Mittal entropy, 2005, Phys. Rev. E 72, 026123.

12. Sparavigna, A.C. Gray-level image transitions driven by Tsallis entropic index, International Journal of Sciences 2015, 4, 16-25.

\begin{tabular}{|c|c|c|c|c|}
\hline $\begin{array}{c}\text { Entropic } \\
\text { indices }(\mathrm{q}, \mathrm{K})\end{array}$ & $\begin{array}{c}\text { Threshold } \\
\text { (T-entropy) }\end{array}$ & $\begin{array}{c}\text { Number of } \\
\text { edge pixels }\end{array}$ & $\begin{array}{c}\text { Threshold } \\
\text { ( } \text {-entropy) }\end{array}$ & $\begin{array}{c}\text { Number of } \\
\text { edge pixels }\end{array}$ \\
\hline 0.01 & 119 & 7807 & 120 & 7901 \\
\hline 0.05 & 119 & 7807 & 120 & 7901 \\
\hline 0.1 & 119 & 7901 & 120 & 7901 \\
\hline 0.15 & 119 & 7901 & 120 & 7901 \\
\hline 0.2 & 120 & 7901 & 120 & 7901 \\
\hline 0.4 & 120 & 7901 & 120 & 7901 \\
\hline 0.6 & 120 & 7901 & 120 & 7901 \\
\hline 0.8 & 120 & 7901 & 120 & 7901 \\
\hline 0.9 & 120 & 7901 & 119 & 7807 \\
\hline 0.99 & 120 & 7901 & 119 & 7807 \\
\hline
\end{tabular}

Fig.1. Lena and the corresponding bi-level black and white image, for threshold 120. The table shows the optimized thresholds obtained using Tsallis (T-entropy) and Kaniadakis ( $\kappa$-entropy) entropies, for several values of entropic indices. In the limit $q \rightarrow 1$, Tsallis entropy provides Shannon result, and for $\kappa \rightarrow 0$, Kaniadakis entropy becomes

Shannon entropy. When the image is segmented in a bi-level black and white image according to the given threshold, the number of edge pixels between black and white regions are calculated. If we assume the "best" bilevel image being that having the largest number of edge pixels, the threshold to choose is 120 from T-entropy and $\kappa$-entropy. 

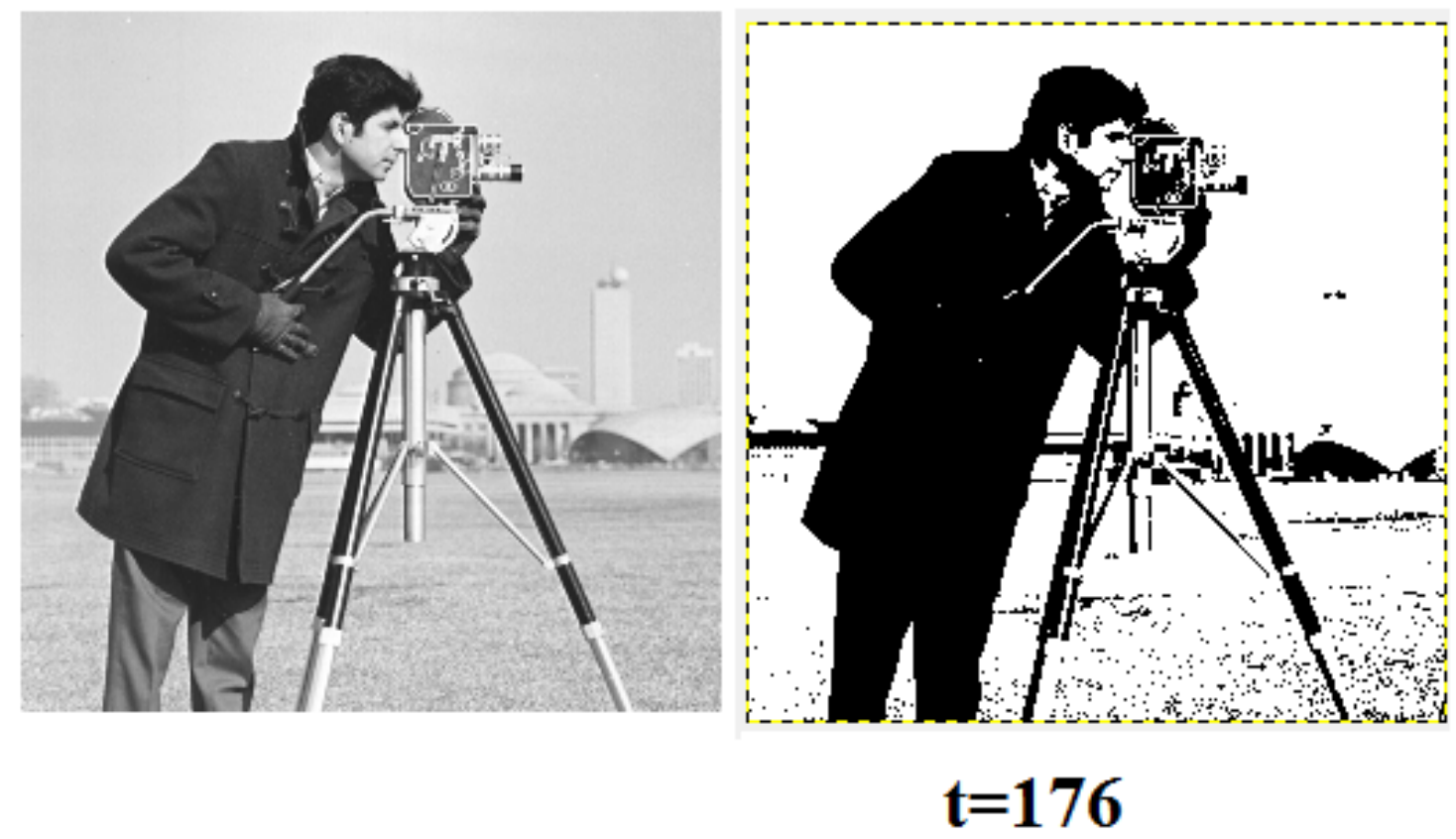

\begin{tabular}{|c|c|c|c|c|}
\hline $\begin{array}{c}\text { Entropic } \\
\text { indices }(\kappa, q)\end{array}$ & $\begin{array}{l}\text { Threshold } \\
\text { (T-entropy) }\end{array}$ & $\begin{array}{l}\text { Number of } \\
\text { edge pixels }\end{array}$ & $\begin{array}{l}\text { Threshold } \\
\text { (K-entropy) }\end{array}$ & $\begin{array}{l}\text { Number of } \\
\text { edge pixels }\end{array}$ \\
\hline 0.01 & 138 & 4559 & 176 & 7186 \\
\hline 0.05 & 140 & 4557 & 175 & 6938 \\
\hline 0.1 & 141 & 4579 & 175 & 6938 \\
\hline 0.15 & 143 & 4596 & 174 & 6734 \\
\hline 0.2 & 145 & 4570 & 171 & 6176 \\
\hline 0.3 & 148 & 4628 & 168 & 5716 \\
\hline 0.4 & 154 & 4695 & 165 & 5323 \\
\hline 0.5 & 160 & 4895 & 160 & 4895 \\
\hline 0.6 & 165 & 5323 & 154 & 4695 \\
\hline 0.7 & 167 & 5570 & 148 & 4628 \\
\hline 0.8 & 171 & 6176 & 145 & 4570 \\
\hline 0.9 & 175 & 6938 & 141 & 4579 \\
\hline 0.99 & 175 & 6938 & 138 & 4559 \\
\hline
\end{tabular}

Fig.2. Cameraman and the corresponding bi-level black and white image, for threshold 176. The table shows the optimized thresholds obtained using T-entropy and $\kappa$-entropy, for several values of entropic indices. Note again, that in the limit $q \rightarrow 1$, Tsallis entropy provides Shannon result, and for $\kappa \rightarrow 0$, Kaniadakis entropy becomes Shannon entropy. As we did for Lena, the image is segmented in a bi-level black and white image according to the given threshold; the number of edge pixels between black and white regions are calculated. If we assume the "best" bilevel image being that having the largest number of edge pixels, the threshold to choose is 176 from $\kappa$-entropy. Note that the best result is corresponding to the Shannon limit 

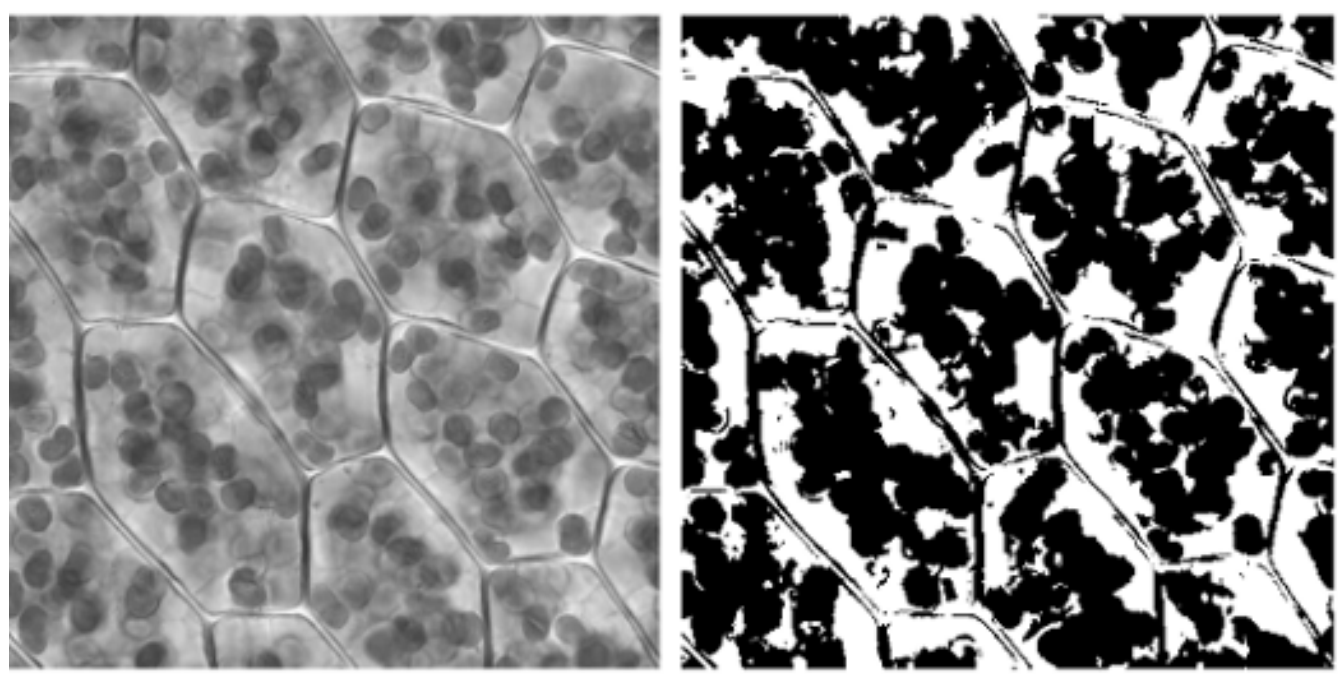

$\mathrm{t}=150$

\begin{tabular}{|c|c|c|c|c|}
\hline $\begin{array}{c}\text { Entropic } \\
\text { indices }(\kappa, \mathrm{q})\end{array}$ & $\begin{array}{c}\text { Threshold } \\
\text { (T-entropy) }\end{array}$ & $\begin{array}{c}\text { Number of } \\
\text { edge pixels }\end{array}$ & $\begin{array}{c}\text { Threshold } \\
\text { ( -entropy) }\end{array}$ & $\begin{array}{c}\text { Number of } \\
\text { edge pixels }\end{array}$ \\
\hline 0.01 & 154 & 20663 & 150 & 21461 \\
\hline 0.05 & 154 & 20663 & 150 & 21461 \\
\hline 0.1 & 155 & 20450 & 150 & 21461 \\
\hline 0.15 & 155 & 20450 & 151 & 21277 \\
\hline 0.2 & 154 & 20663 & 151 & 21277 \\
\hline 0.3 & 154 & 20663 & 151 & 21277 \\
\hline 0.4 & 154 & 20663 & 153 & 20903 \\
\hline 0.5 & 153 & 20903 & 153 & 20903 \\
\hline 0.6 & 153 & 20903 & 154 & 20663 \\
\hline 0.7 & 151 & 21277 & 154 & 20663 \\
\hline 0.8 & 151 & 21277 & 154 & 20663 \\
\hline 0.9 & 151 & 21277 & 155 & 20450 \\
\hline 0.99 & 150 & 21461 & 154 & 20663 \\
\hline
\end{tabular}

Fig.3. Microscopic image of cells (courtesy Kristian Peters, Wikipedia) in gray-level rendering and the corresponding bi-level image. The table is showing the optimized thresholds obtained using T-entropy and $\kappa$ entropy. As we did for Lena and Cameraman, the image is segmented in a bi-level black and white image according to the given threshold; the number of edge pixels between black and white regions are calculated. If we assume the "best" bi-level image being that having the largest number of edge pixels, the threshold to choose is 150 from T- and $\kappa$-entropies. Again, the best result corresponds to the Shannon limit. 


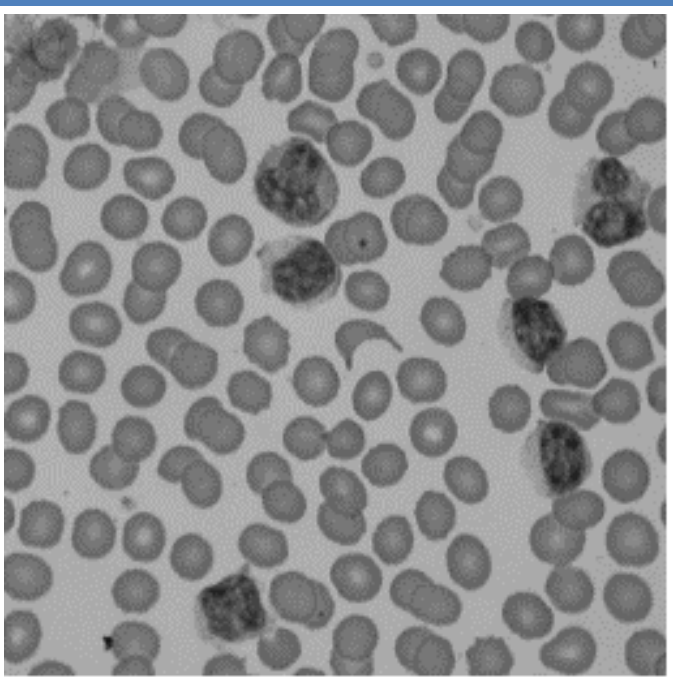

Blood,

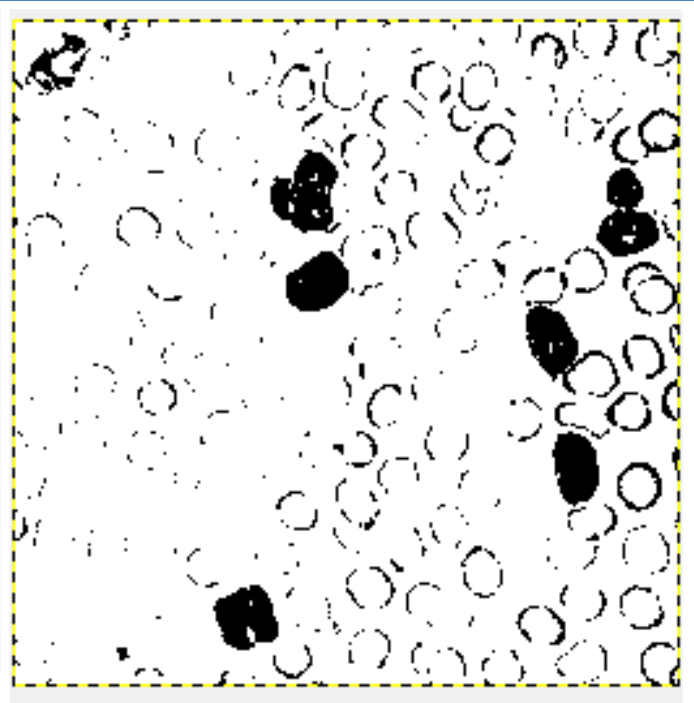

$\mathbf{t}=\mathbf{1 0 2}$

\section{Sample 1}

\begin{tabular}{|c|c|c|c|c|}
\hline $\begin{array}{c}\text { Entropic } \\
\text { indices }(\kappa, q)\end{array}$ & $\begin{array}{c}\text { Threshold } \\
\text { (T-entropy) }\end{array}$ & $\begin{array}{c}\text { Number of } \\
\text { edge pixels }\end{array}$ & $\begin{array}{c}\text { Threshold } \\
\text { (к-entropy) }\end{array}$ & $\begin{array}{c}\text { Number of } \\
\text { edge pixels }\end{array}$ \\
\hline 0.01 & 102 & 7215 & 93 & 1525 \\
\hline 0.05 & 98 & 3253 & 93 & 1525 \\
\hline 0.1 & 96 & 2281 & 94 & 1735 \\
\hline 0.2 & 95 & 1966 & 94 & 1735 \\
\hline 0.3 & 95 & 1966 & 95 & 1966 \\
\hline 0.5 & 95 & 1966 & 95 & 1966 \\
\hline 0.7 & 95 & 1966 & 95 & 1966 \\
\hline 0.8 & 94 & 1735 & 95 & 1966 \\
\hline 0.9 & 94 & 1735 & 96 & 2281 \\
\hline 0.99 & 93 & 1525 & 102 & 7215 \\
\hline
\end{tabular}

Fig.4. Microscopic image of blood cells (courtesy Wikipedia) and the table showing the optimized thresholds obtained using T-entropy and $\kappa$-entropy. If we assume the "best" bi-level image being that having the largest number of edge pixels, the threshold to choose is 102 from $\mathrm{T}$ - and $\kappa$-entropies. Note that, in this case, the best result is quite different from the Shannon limit. It is at the opposite end of the interval spanned by the entropic index. 


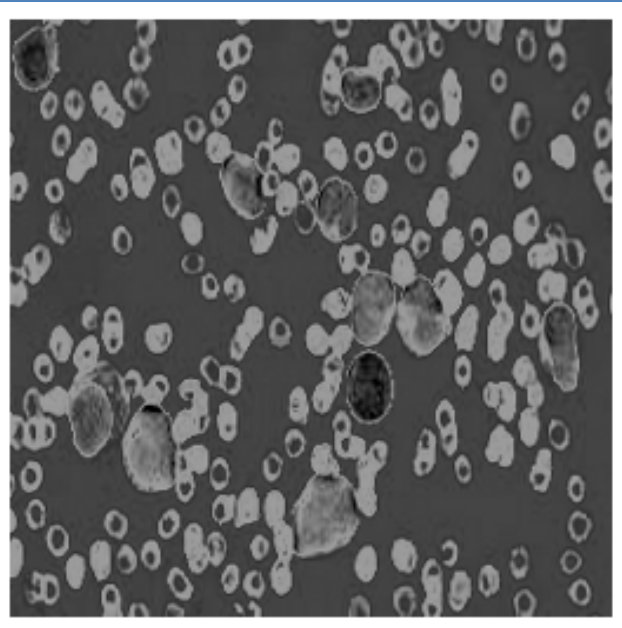

Blood,

Samples 2

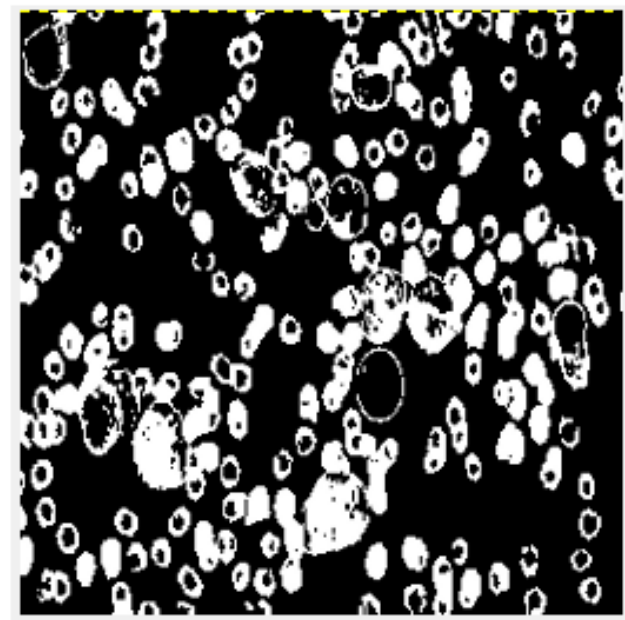

$\mathbf{t}=96$

\begin{tabular}{|c|c|c|c|c|}
\hline $\begin{array}{c}\text { Entropic } \\
\text { indices }(\kappa, \mathrm{q})\end{array}$ & $\begin{array}{c}\text { Threshold } \\
\text { (T-entropy) }\end{array}$ & $\begin{array}{c}\text { Number of } \\
\text { pixels at edges }\end{array}$ & $\begin{array}{c}\text { Threshold } \\
\text { ( } \text {-entropy) }\end{array}$ & $\begin{array}{c}\text { Number of } \\
\text { pixels at edges }\end{array}$ \\
\hline 0.01 & 90 & 13561 & 49 & 858 \\
\hline 0.05 & 90 & 13561 & 49 & 858 \\
\hline 0.1 & 91 & 13603 & 49 & 858 \\
\hline 0.2 & 91 & 13603 & 49 & 858 \\
\hline 0.3 & 96 & 13691 & 49 & 858 \\
\hline 0.4 & 96 & 13691 & 49 & 858 \\
\hline 0.5 & 87 & 13530 & 87 & 13530 \\
\hline 0.55 & 49 & 858 & 87 & 13530 \\
\hline 0.6 & 49 & 858 & 96 & 13691 \\
\hline 0.7 & 49 & 858 & 96 & 13691 \\
\hline 0.8 & 49 & 858 & 91 & 13603 \\
\hline 0.9 & 49 & 858 & 91 & 13603 \\
\hline 0.99 & 49 & 858 & 90 & 13561 \\
\hline
\end{tabular}

Fig.4. Another microscopic image of blood cells (courtesy Wikipedia) and the table showing the optimized thresholds obtained using T-entropy and $\kappa$-entropy. If we assume the "best" bi-level image being that having the largest number of edge pixels, the threshold to choose is 96 from $\mathrm{T}$ - and $\kappa$-entropies. Note that, in this case again, the best result is different form the Shannon limit. It is about the values 0.6 and 0.7 of the $\kappa$ entropic index, and about 0.3 and 0.4 of the $q$ index. In fact, here we are observing, as the index is spanning the interval $(0,1)$, an "image transition", which is accompanied by a texture transition in it [12]. 